\title{
Challenges in Administering a Clinical Trials Registry: Lessons from the Clinical Trials Registry-India
}

\author{
Arvind Pandey • Abha Aggarwal • Mohua Maulik • \\ Jyotsna Gupta · Atul Juneja
}

Published online: 16 March 2013

(C) Springer International Publishing Switzerland 2013

\begin{abstract}
The Clinical Trials Registry - India (CTRI), an online system (www.ctri.nic.in) for the registration of clinical trials being conducted in India, has its secretariat at the National Institute of Medical Statistics of the Indian Council of Medical Research in New Delhi. The primary objective of the CTRI is to ensure that all clinical trials conducted in India are registered in order to bring transparency, accountability and access to clinical trials. Since its launch on 20 July 2007, the CTRI has gone from strength to strength and, as of January 2013, more than 3,300 trials had been registered. Although initiated as a voluntary exercise, registration of trials requiring approval by the drug regulatory authority in India has been made mandatory. Editors from 11 major biomedical journals in India require submission of the clinical trial registration number as a prerequisite for publication. In addition, several ethics committees also insist upon trial registration. The CTRI is a primary registry of the WHO's International Clinical Trials Registry Platform. This article discusses the challenges encountered during the setting up of the CTRI and the strategies adopted, and also explains the steps to trial registration in the CTRI.
\end{abstract}

\section{Introduction}

The Clinical Trials Registry - India (CTRI), launched on 20 July 2007, is Asia's first clinical trials registry (www. ctri.nic.in) [1]. The CTRI not only registers trials being conducted in India, but also accepts trials from countries that do

A. Pandey $(\bowtie)$ - A. Aggarwal · M. Maulik · J. Gupta .

A. Juneja

Clinical Trials Registry - India, National Institute of Medical

Statistics, Indian Council of Medical Research, New Delhi

110029, India

e-mail: arvindpandey@icmr.org.in not have a primary registry of their own [2-4]. The initiative gained a major boost with the implementation of mandatory registration of clinical trials requiring approval by the Indian drug regulatory authority [Drugs Controller General (India), DCGI] [5]. As not all trials require approval by the DCGI, ethics committees and journal editors of the Indian biomedical journals have been alerted to its existence and purpose in an attempt to bring the other trials under the umbrella of the CTRI through their influence. With increased awareness, the CTRI is becoming a valuable and informative database for a wide range of stakeholders, including patients, researchers, activists and Parliamentarians [6]. As of January 2013, more than 3,300 trials had been registered in the CTRI, and being a primary registry of the WHO's International Clinical Trials Registry Platform (ICTRP), the CTRI is a significant contributor to the global pool of accessible clinical trial data.

Trial registration in the CTRI is a paperless and online procedure. Based on experiences gathered from trial registrations and feedback from stakeholders, the CTRI software application has been upgraded, and the revised version was activated on 15 March 2011. While the benefits, advantages and drawbacks of the CTRI have been discussed elsewhere $[6,7]$, this article attempts to describe the challenges encountered during the setting up of the CTRI and the strategies adopted to overcome these. In addition, the article highlights the common problems from the registrant's perspective to enable smooth and quick trial registration.

\section{Challenges in Setting Up the CTRI}

\subsection{Trials Accepted for Registration}

Initially in the CTRI, in keeping with the mandate set by the WHO, only interventional trials where the first patient 
had yet to be enrolled were registered (prospective registration). A total of 11 trials were registered in the first 6 months. Subsequently, registration of ongoing and completed trials (retrospective registration) was allowed as there was significant lack of awareness among trialists regarding trial registration and to create a comprehensive database of clinical trials.

In addition, terminated trials, which have been granted DCGI and/or ethics committee approval, but have not been conducted in the country for some reason or other, are also registered in the CTRI. Upon registration, trials are immediately flagged according to the status at the time of registration (Fig. 1). Postmarketing surveillance studies as well as observational and bioavailability and bioequivalence $(\mathrm{BA} / \mathrm{BE})$ studies are also being registered.

Currently, it is not mandatory to register observational studies; however, if submitted, these trials are registered in the CTRI. Despite the added workload, observational studies are considered for registration in the CTRI, on the premise that many researchers are not aware of the difference between 'observational studies' and 'interventional trials.' Also, there have been instances where researchers have classified interventional trials as 'observational' simply because they were 'observing' the effect of drug X on blood pressure.

At present, the data set fields for all these studies are identical, although for observational studies, the intervention and comparator agent section (an otherwise compulsory field) is to be left blank. Mandatory registration of $\mathrm{BA} / \mathrm{BE}$ trials is under active consideration, and the data set fields may need to be tweaked for registration of these trials.

\subsection{Deciding Data Set Fields}

The WHO originally identified 20 data set fields to be declared prior to trial registration, and additional fields have been added to this in the CTRI (Table 1). This has been done in view of certain requirements unique to the Indian scenario, to enable trial validation and streamline data collection, retrieval and analysis.

\subsubsection{Trials as Part of Post-Graduation Theses}

Clinical trials being conducted as part of post-graduation (PG) theses are encouraged to be registered in the CTRI via dissemination workshops and presentations at medical colleges across the country. Ethics committees are requested to recommend registration of such studies. This move is expected to help in preventing duplication of research as most of these data are not published. Further, it would help raise the standard of research in the country. To differentiate between clinical trials per se and those being conducted as part of a PG thesis, the question "Is this part of a post-graduation thesis?" (yes or no) is now a part of the data set form.

\begin{tabular}{|c|c|c|}
\hline & & \\
\hline CTRI Number & & CTRI/2012/12/003217 [Registered on: 13/12/2010 Trial Registered Prospectively \\
\hline Last Modified On: & & $12 / 12 / 2012$ \\
\hline Post Graduate Thes & & No \\
\hline Type of Trial & & Observational \\
\hline Type of Study & & Cohort Study \\
\hline Study Design & & Other \\
\hline FULL DETAILS (Read & 1 -only) - & $\rightarrow$ Click Here to Create PDF for Current Dataset af mal \\
\hline CTRI Number & CTRI/2 & 012/12/003260 [Registered on: 26/12/2012] Trial Registered Retrospectively \\
\hline Last Modified On: & $24 / 12 / 2$ & 2012 \\
\hline $\begin{array}{l}\text { Post Graduate } \\
\text { Thesis }\end{array}$ & No & \\
\hline Type of Trial & PMS & \\
\hline Type of Study & Drug & \\
\hline Study Design & Other & \\
\hline FULL DETAILS (Read & $1-$ only) - & $\rightarrow$ click Here to Create PDF for Current Dataset of \\
\hline CTRI Number & & CTRI/2011/12/002283 [Registered on: 23/12/2011 Terminated Trial Registered \\
\hline Last Modified On: & & $23 / 12 / 2011$ \\
\hline Post Graduate Thesi & & No \\
\hline Type of Trial & & Interventional \\
\hline Type of Study & & $\begin{array}{l}\text { Drug } \\
\text { Biological }\end{array}$ \\
\hline Study Design & & Other \\
\hline
\end{tabular}

Fig. 1 Trials flagged (encircled in red) upon registration according to recruitment status (screen shot of three registered trials) 
Table 1 Data set fields of the CTRI

\begin{tabular}{ll}
\hline Part 1 & \\
1 & Public title of study \\
2 & Scientific title of study \\
Part 2 & \\
1 & Secondary IDs \\
2 & $\begin{array}{l}\text { Principal investigator or overall trial } \\
\text { coordinator (multicenter study) details* }\end{array}$ \\
3 & Contact person (scientific query) \\
4 & Contact person (public query)
\end{tabular}

\section{Part 3}

1

2

3

4

\section{Part 4}

$1 \quad$ Site(s) of study*

2

3

4

Part 5

1

\section{Part 6}

1

2

3

4

5

Part 7

1 Primary outcome(s)

2 Secondary outcome(s)

3 Target sample size

- Total

- India*

$4 \quad$ Phase of trial*

5 Date of first enrollment

- India*

- Global

6

Part 8

\begin{tabular}{ll}
1 & Recruitment status of trial \\
& $\bullet$ Global status \\
& - India status* \\
3 & Publication details* \\
& Brief summary* \\
\hline
\end{tabular}

Additional fields of the CTRI are marked with an asterisk

\subsubsection{Site Address}

In view of the increasing number of trial-related queries from interested patients and their relatives, the name and contact details of Indian investigators, complete addresses of trial sites, and email address and phone numbers are recorded. This is expected to help interested trial participants contact the trial site and trial principal investigators with ease.

\subsubsection{India-Specific Details}

For multi-country or global trials, information regarding the India-specific target sample size, date of first enrollment and recruitment status of the trial is captured in the main data set form alongside the global information in distinct columns earmarked for the purpose. The reasons for this have been discussed elsewhere [8].

\subsection{Trial Verification and Validation}

As recommended by the WHO, all clinical trials prior to registration should be 'verified and validated.' In the initial stages of setting up the registry, there were no clear-cut guidelines to achieve this, i.e., establish the authenticity of the individual submitting a trial and confirm that a trial was actually being conducted. Various possibilities, such as identification of nodal officers at various institutes to aid the verification and validation process, were explored but were found to be unsatisfactory because of the cumbersome and time-consuming nature of the procedure.

After much deliberation, submission of ethics committee and DCGI approval (if applicable) was identified as a holistic tool for validating a trial. Currently, submission of these documents is essential for trial registration in the CTRI.

\subsubsection{Ethics Approval}

As all clinical trials are bound to obtain ethics approval, this document was identified as a key trial validation tool. The submitted ethics approval document is cross-checked with the trial data set form for the following:

- The scientific title exactly matches that cleared by the ethics committee (EC). In case of discrepancies, corrected versions are to be submitted.

- The name of the principal investigator and site matches that in the EC approval.

- Validity of EC approval.

- Incorporation of EC conditions, if any. 
To encourage prospective trial registration and avoid delay in trial initiation, for multicentric trials, submission of EC approval from one site is considered to be adequate for trial registration. Subsequently, EC approvals for other participating sites are expected to be uploaded as and when available. Submission entails uploading of scanned copies of EC approvals in the CTRI data set form. These files are not available in the public domain.

\subsubsection{DCGI Approval}

The DCGI approval (if applicable) is another valuable tool for validating a clinical trial, and this document is crosschecked with the trial data set form for the following:

- The protocol number, including the version and date, matches that provided in the secondary ID section of the form.

- Incorporation of DCGI conditions, if any.

As mentioned above, upload of DCGI approval (if applicable) is mandatory prior to trial registration. It is the responsibility of the registrant or sponsor to establish whether DCGI approval is applicable for a trial or not.

\subsubsection{Third Party Validation}

In addition to the above, third party validation is ensured by establishing contact with trial contact persons through email (preferable) or telephone (only in rare instances where there is no email facility). All contact persons should be from India, including for trials being conducted in India as part of global clinical trials. Trial registration is undertaken only after an email response has been received from the overall trial coordinator (if applicable) and contact person (scientific and public queries). Verification mails are also sent to site contact persons (site investigators), and again, to avoid delay in patient recruitment, trial registration is undertaken even if one investigator (at site) confirms participation.

\subsection{Enhancing Awareness and Ensuring Compliance}

Several active measures were taken and continue to be taken to increase awareness and enhance trial registration. In 2008, the CTRI organized a workshop for editors of several Indian biomedical journals. This resulted in a statement being issued by editors of 11 major Indian biomedical journals, declaring that only registered trials would be considered for publication [9]. This statement was in addition to and distinct from the statement published by the International Committee of Medical Journal Editors in 2004 [10], and helped to provide a much needed boost to this initiative.

Appreciating the benefits of trial registration, the office of the DCGI, the drug regulatory body in India, made it mandatory for trials requiring DCGI permission to be registered in the CTRI prospectively. This was implemented with effect from 15 June 2009 [5]. This had a further positive and significant impact on trial registration.

Ethics committees are a key link between the trialist and the CTRI. They also have been sensitized, and as a result several ethics committees now advocate registration as a pre-condition to trial approval.

Moreover, numerous CTRI workshops and advocacy meetings have been conducted across the country to increase awareness among researchers and the pharmaceutical industry.

\subsection{Audit Trail}

Audit trail has been implemented to capture the changes made to the trial data set after a trial has been registered. As a result, all changes made to a registered trial's data are available in the public domain as well. In addition, major changes in the data set form, such as sample size, primary and secondary outcome measures, etc., require submission of the ethics committee and DCGI (if applicable) approval.

\subsection{Registrant's Account Management}

As site surfing, including searching and viewing of registered trials, does not require the creation of an account, registration of new applicants is restricted to only those who wish to register a clinical trial. Furthermore, in view of the increasing number of overseas requests, registration of international registrants is also allowed.

Due to the attrition in the pharmaceutical industry and inadvertent creation of multiple user accounts by a single user, a need was felt for the facility to transfer trials between registrants. In the revised version, it is now feasible to transfer a trial from one registrant to another, within a company or between companies, upon submission of appropriate authorization documents.

It was observed that a single user could create multiple accounts for a variety of reasons (such as loss of a password), which in turn led to difficulties in both administering and managing trial data by the user. To circumvent this issue, the email address used for registering as a new applicant has been made unique, i.e., only one user account may be created for a particular email address.

\section{Bottlenecks to Trial Registration}

\subsection{Registrant's Profile}

The first bottleneck to trial registration is the point of contact (i.e., the registrant) between the sponsor and the 
CTRI. Speed and ease of trial registration are crucially dependent upon the registrant's comprehension capabilities, compliance and response time to CTRI requests for trial-related modifications and queries, if any. More than 40 trials remain pending with the CTRI because of noncompliance with requests for modifications and nonresponse to repeated mails sent for clarification of issues/ problems being encountered in incorporating requested changes.

For quick and smooth trial registration, it is of utmost importance that while creating an account, the contact details (including email ID) of the specific designated registrant be provided. More often than not, an account is opened in the name of a very senior official from the concerned organization while practically the account is operated by a junior official. As all trial-related communications (manual and automated) are made to the registered email address, these do not make sense (or are not of priority) to the receiver (senior official), while the junior official who has submitted the trial is not kept in the loop regarding the status and queries associated with the trial, thereby leading to miscommunication and subsequent delay in trial registration.

If a registrant leaves the organization or is no longer part of the trial registration team, all trials may be assigned to another registrant by simply updating the contact details available under Edit Profile.

\subsection{Delayed Trial Submission}

Trials submitted to the CTRI are reviewed, and registrants often fail to factor in the processing time. If prospective trial registration is desired, trials should be uploaded and submitted for review even before requisite approvals have been obtained. After completion of all necessary formalities, i.e., third party validation and trial data set review and modifications as per CTRI requirements, the trial is kept pending until submission of the necessary approvals. Subsequently, upon submission of approvals, a trial can be registered without much delay.

\subsection{Steps of Trial Registration}

As mentioned earlier, trial registration in the CTRI is a purely online process. There have been instances where registrants have downloaded a copy of the CTRI data set description form, which is meant as a reference guide only, and filled in the form with trial details and submitted a copy by email or post. Such submissions are not accepted in the CTRI.

The CTRI data set form is divided into eight parts, where each part may have more than one sub-part (Table 1). Filling in of part 1 is compulsory for a trial to be 'created,' and subsequently other parts can be completed in any order at the convenience of the registrant. After filling

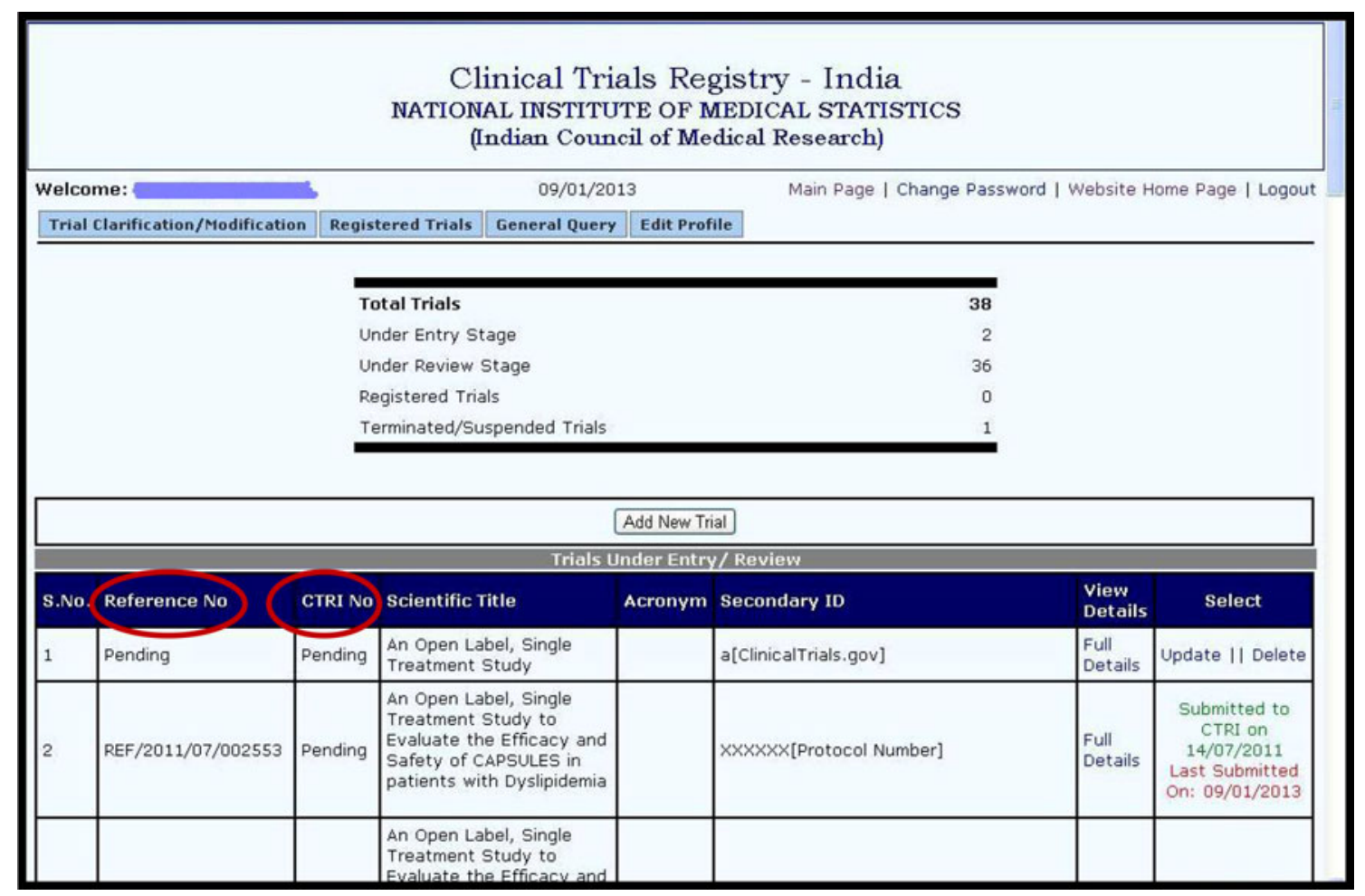

Fig. 2 Welcome page of the registrant in the CTRI displaying trials under the Entry (both REF and CTRI number pending) and Review (only CTRI number pending) stages 
Fig. 3 Algorithm for trial registration in CTRI

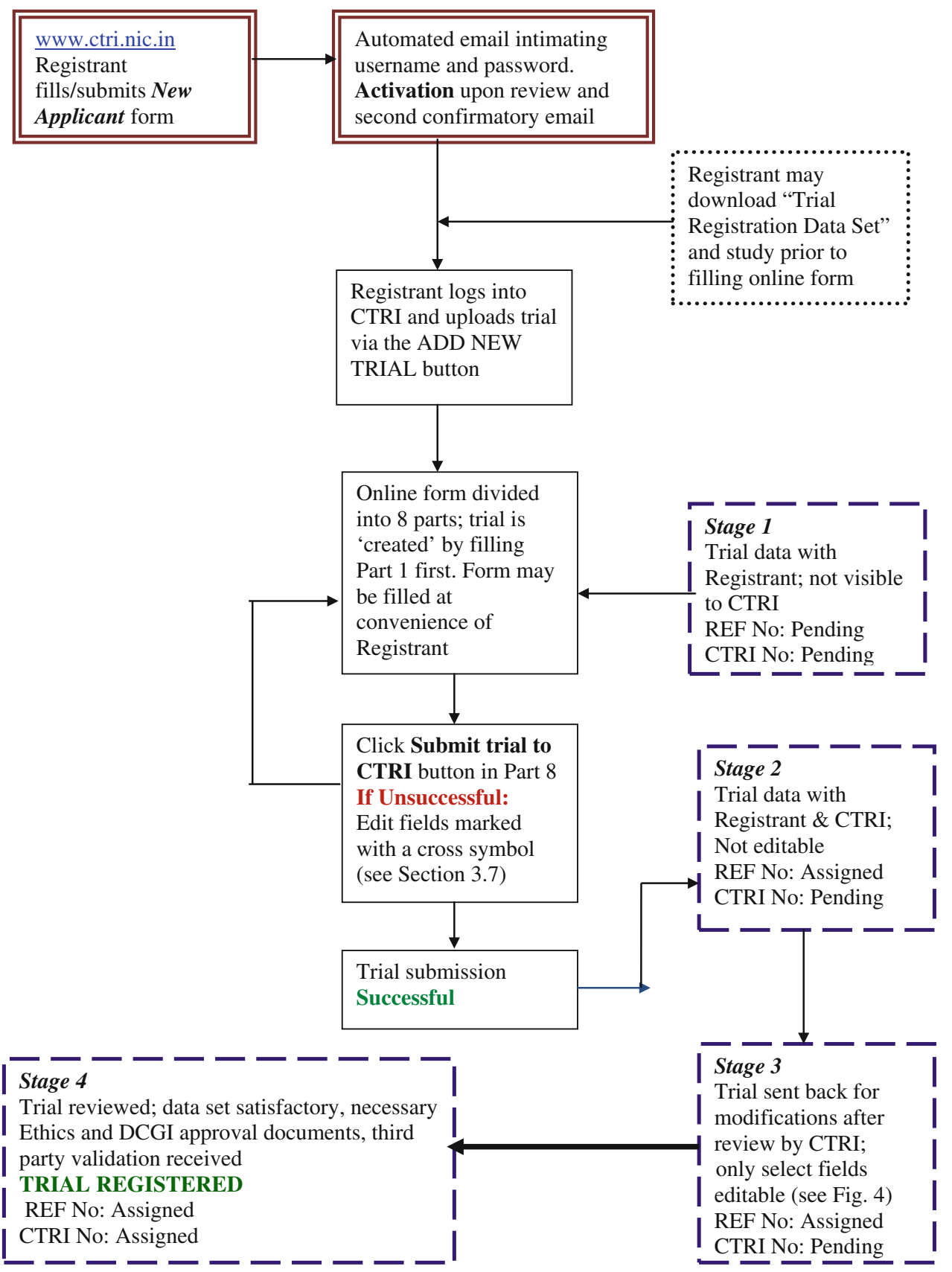

in part 1 , the trial is 'created' and appears on the registrant's welcome page, with both REF and CTRI numbers as pending (discussed subsequently in Sect. 3.5). The full data set form can be viewed by clicking on view full details, while the data set form can be completed via the update button. Context information regarding what each field signifies and what information is to be uploaded is available against each data set field and can be referred to by clicking the icon present alongside each data set field.

Many registrants complain of no response to trials submitted apparently months ago simply because they did not hit the 'submit trial to CTRI' button. If on the welcome page the trial shows the REF as 'pending,' this indicates that the trial has yet to be submitted to the CTRI, and if only the CTRI number is 'pending,' this indicates that the trial has not yet been registered (Fig. 2).

The stages of a trial and the process of trial registration in the CTRI are further described below (Fig. 3):

- Stage 1: a trial for registration is 'created' by the registrant and the data set form partially or completely filled online. At this stage, the trial is visible only to the registrant (but not to the CTRI), and both the acknowledgement number (REF number) and registration number (CTRI number) are indicated as 'pending.' 
- Stage 2: the trial is submitted to the CTRI and an REF number assigned to the trial. A trial in this stage is viewable to the registrant and CTRI, but is not editable. The CTRI number is still 'pending.'

- Stage 3: the trial is reviewed by the CTRI, and only fields requiring modifications and/or clarifications are unlocked for editing by the registrant and the trial is sent back to the registrant. In case any other field needs to be edited, the registrant can send a request for unlocking of the desired field(s). At this stage, the CTRI number is still 'pending.' A trial can be sent back to the registrant any number of times until all concerns are satisfactorily addressed. Each time, an automated mail alert is sent to the registrant regarding the need for action on a trial.

- Stage 4: upon resubmission of the trial, subsequent to satisfactory trial data set modifications, receipt of third party validation, necessary ethics committee and DCGI approval documents and their scrutiny, the trial is registered, and a CTRI number is assigned to the trial.

\subsection{Preview before Trial Submission}

Once a trial has been submitted to the CTRI, all fields become locked (i.e., un-editable) unless some clarification is desired. In order to update any field, a request for field unlocking can be sent to the CTRI for release of trial data set field(s) for correction of oversight errors on the part of the registrant. This necessitates a trial to be sent back to the registrant for rectifications, resulting in delay of trial processing and subsequent registration. Hence, to ensure submission of correct trial details (as well as check for typographical errors), a PDF file of the data set form should be generated (link available in part 8) and circulated among all concerned personnel to verify trial details before submission of trial to the CTRI. This helps reduce errors in the uploaded data and time taken to register a trial.

\subsection{Likely Errors in Trial Submission}

A trial will not be successfully submitted unless all (with certain exceptions as mentioned below) data set fields have been filled in, and an error message highlights the data set field(s) requiring further attention. The fields, which are optional (i.e., may be left blank), include the principal investigator or overall trial coordinator, method of generating the randomization sequence, method of allocation concealment and blinding/masking (Table 1).

Upon trial submission, a successful trial submission message is generated along with an acknowledgement number. All trial-related queries must be accompanied by the acknowledgement number beginning with the REF or, if registered, the CTRI number.
The following are the fields where registrants commonly encounter errors during trial submission:

\subsubsection{Intervention and Comparator Agent}

Information regarding the intervention and comparator agent are to be incorporated by clicking on add new and selecting the type as intervention or comparator agent, as applicable.

Unless details of both the intervention and comparator agent have been provided, a trial will not be submitted successfully. Therefore, single arm trials should mention 'not applicable' under comparator agent to enable successful trial submission. However, observational studies can be submitted without either intervention or comparator agent details.

\subsubsection{Number of Sites of Study and Ethics Committee}

Number of study sites should match the number in the EC approval status given (unless the response to is there an independent ethics committee? is yes).

It should be mentioned here that in India there are two types of ethics committees, namely institutional ethics committees and independent or non-institutional ethics committees [8]. The former are affiliated with a particular institution (although functioning remains "independent") and review only trials being conducted at that particular institution. Independent or non-institutional ethics committees are not associated with any institution and hence are termed "independent" ethics committees. One independent ethics committee can approve the conduct of a trial at more than one site.

\subsubsection{Monetary/Material Support}

The less discerning registrant fails to differentiate between monetary/material support and the subsequent field, that of the primary sponsor. These are two distinct data set fields, and they need to be filled in separately. If there is no monetary/material support, 'nil' or 'none' should be written. However, for an institute- or hospital-based trial, this should be acknowledged in this section.

\subsection{Review of Trial Data by CTRI}

Once a trial has been "submitted," the trial cannot be edited by the registrant but is viewable. Trial data are carefully scrutinized by CTRI scientists for adherence to data set requirements and clarity of the information presented. It can be mentioned here that while trial registration may be mandatory or voluntary (for trials not coming under the purview of the DCGI), if a trial is 


\begin{tabular}{|c|c|c|c|}
\hline \multicolumn{4}{|c|}{ Part 3} \\
\hline 1 & Source/s of monetary or material support & No Clarification Sought & Field Locked \\
\hline 2 & Primary sponsor & No Clarification Sought & Field Locked \\
\hline 3 & Secondary Sponsor & No Clarification Sought & Field Locked \\
\hline 4 & Countries of recruitment & No Clarification Sought & Field Locked \\
\hline \multicolumn{4}{|c|}{ Part 4} \\
\hline 1 & Site/s of study & Clarification Sought & Modif \\
\hline 2 & Name of Ethics Committee and approval status & No Clarificatium sudgith & Modify \\
\hline 3 & Regulatory clearance obtained from DCGI & No Clarification Sought & Field Locked \\
\hline 4 & Health condition/problems studied & No Clarification Sought & Field Locked \\
\hline \multicolumn{4}{|c|}{ Part 5} \\
\hline 1 & Study Type & Clarification Sought & Modify \\
\hline \multicolumn{4}{|c|}{ Part 6} \\
\hline 1 & Intervention and comparator agent & No Clarification Sought & Field Locked \\
\hline 2 & Inclusion \& Exclusion Criteria & No Clarification Sought & Field Locked \\
\hline 3 & Method of generating randomization sequence & No Clarification Sought & Field Locked \\
\hline 4 & Method of allocation concealment & No Clarification Sought & Field Locked \\
\hline 5 & Blinding/masking & No Clarification Sought & Field Locked \\
\hline \multicolumn{4}{|c|}{ Part 7} \\
\hline 1 & Primary outcome/s & No Clarification Sought & Field Locked \\
\hline 2 & Secondary outcome/s & No Clarification Sought & Field Locked \\
\hline 3 & Target sample size & No Clarification Sought & Field Locked \\
\hline 4 & Phase of trial & No Clarification Sought & Field Locked \\
\hline 5 & Date of first enrollment & No Clarification Sought & Field Locked \\
\hline 6 & Estimated duration of trial & No Clarification Sought & Field Locked \\
\hline \multicolumn{4}{|c|}{ Part 8} \\
\hline 1 & Recruitment status of trial & No Clarification Sought & Modi \\
\hline 2 & Brief Summary & No Clarification Sought & Field Locked \\
\hline \multirow[t]{2}{*}{3} & Publication & No Clarification Sought & Field Locked \\
\hline & & & Submit to CTRI \\
\hline
\end{tabular}

Fig. 4 View of the trial data set form received by the registrant after review by the CTRI

uploaded with the specific purpose of being registered, voluntarily or otherwise, the CTRI requirements are mandatory and have to be fulfilled if a trial is to be registered.

After review, the trial can be sent back in case any changes or additional information is desired. While efforts are made to ensure that all clarifications are sought in the first round itself, oversight and non-availability of approval documents often necessitate further rounds of clarifications and modifications. In addition, registrants also commonly fail to address all clarifications sought and click on the submit to CTRI button without checking if all desired modifications have been incorporated or satisfactorily addressed.

\subsection{Data Set Fields Commonly Requested for Modifications}

As mentioned previously, once a trial has been reviewed by CTRI scientists, only the data set fields that need clarifications or modifications will be modifiable, while the others will be locked for editing. The exceptions to this include the recruitment status of trial and the name of ethics committee and approval status, which remain permanently unlocked, even upon trial registration. These fields remain unlocked to enable upload of pending ethics approvals and update of trial status in a timely manner (Fig. 4).

The data set fields that most often require modifications include:

\subsubsection{Public Title of Study}

The public title of the study (item 9 of the WHO's trial registration data set) is meant for the lay public and hence should be in simple language. Technical terminologies should be avoided wherever feasible. Alternatively, these can be supplemented by simplified terms, within parentheses.

Yet registrants often submit a public title that is a repetition of the scientific title or insist upon the one that has been submitted to another registry for the purpose of "maintaining uniformity across registries." Others presume 'brevity' to be synonymous with 'simplify.'

\subsubsection{Scientific Title of Study}

The scientific title of the trial should be the same as that approved by the ethics committee. In case of multicentric trials, all ethics approvals should carry the same title, and discrepancies, if any, should be checked before submission of approval documents. 


\subsubsection{Trial Acronym}

Only if a specific trial acronym has been assigned to a trial should it be included in the column provided for this purpose. The field can be left blank if there is no specific acronym. Registrants often include protocol numbers and acronyms for disease conditions as the trial acronym.

\subsubsection{Secondary IDs}

A secondary ID of a trial is any other number that is associated with a trial, such as the protocol number or any other primary registry number, if registered, such as http://ClinicalTrials.gov, ANZCTR, ISRCTN, etc. There is no limit to the number of secondary ID numbers that can be provided. Each secondary ID should be uploaded separately by clicking on add new. Registrants often add multiple secondary IDs in the same column, making differentiation between IDs confusing. Others fail to include other registry numbers and/or protocol numbers, necessitating data set modifications.

It is important to ensure that the complete protocol number, including version and date, which matches that in the DCGI approval document, is incorporated.

\subsubsection{Contact Persons}

All contact persons (scientific and public queries) as well as the principal investigator or overall trial coordinator (optional field) must be from India. This is applicable even for global trials. The only exception is when the trial does not have any site in India, i.e., being conducted in a country that has no primary registry of its own.

In these data set fields, the column 'designation' has been found to be perplexing, particularly for the international registrant. Designation refers to the position at which the contact person is employed in his/her organization.

\subsubsection{Secondary Sponsor}

Trials being sponsored by global pharmaceutical industries operate in India through their Indian counterpart or contract research organization (CRO). In such cases, the DCGI approval is addressed to the Indian agency or CRO, and this should be acknowledged as the secondary sponsor section.

\subsubsection{Intervention and Comparator Agent}

To ensure complete transparency and clarity, the specific name of the intervention(s) and the comparator or control agents being studied is expected to be provided. The international non-proprietary name along with brand/trade names should be mentioned. For an unregistered drug, the generic name, chemical name or company serial number is acceptable. If the intervention consists of several separate treatments (i.e., combination therapy), these should all be listed separated by commas (e.g., "low-fat diet, exercise"). However, different arms of a trial should be entered separately by clicking on add new.

Also, for each intervention, the comparator agent, details such as dosage, frequency, route of administration and total duration of therapy are to be specified. This is a general indicator of the kind of information expected, and appropriate information is to be provided for non-drug trials.

\subsubsection{Site Address Details}

As mentioned in Sect. 2.2.2, the complete addresses of trial sites along with the email address and phone numbers of site principal investigators (PIs) are recorded. Ensuring registrant compliance with this requirement is rather poor, especially when the registrant is based outside India, leading to delays in trial registration.

\subsubsection{Name of Ethics Committee and Approval Status}

The CTRI data set form collects information on the type of ethics committee, i.e., independent or institutional (discussed earlier). Particular emphasis is placed on the name of the ethics committee to enable unambiguous identification of ethics approval with the site of the study [8].

As mentioned earlier (Sect. 3.2), trials can be submitted to the CTRI for processing prior to approval by either the EC or the DCGI. The status should be indicated as submitted/under review or awaited, respectively. Trial registration is, however, undertaken only upon submission of ethics committee and DCGI approvals, wherever applicable.

Upon receipt of approval, scanned copies are to be uploaded after changing the status to approved/obtained. To avoid delay in trial registration, scanned copies of approvals should be uploaded strictly according to instructions and viewed before submission.

The trial review and registration process is briefly summarized in Fig. 5.

\section{Summary}

Several challenges remain from the administrator's perspective, foremost being compliance and adherence to CTRI requirements. Particular emphasis is laid on the quality of information being submitted for registration, and painstaking efforts are made to ensure that there is transparency and clarity with regard to the clinical trial-related 
Fig. 5 Schema for trial review and registration in the CTRI
Trial submitted to CTRI

$\checkmark \quad$ Trial data set details satisfactory

$\checkmark$ Ethics/DCGI (if applicable) approvals match form

$\checkmark$ Verification mails received Clinical Trial Registered

information being provided, often resulting in numerous rounds of clarifications and modifications. Communication gaps, comprehension capabilities, assigning responsibility of trial registration to a novice and lack of commitment to transparency are major causes of delays in trial registration.

Registrants are advised to familiarize themselves with the CTRI's specific requirements to obtain a registration number in the minimum possible time. Registrants are expected to maintain a continuous active channel of communication with the CTRI even after trial registration has been completed to provide updates pertaining to protocol amendments, changes in contact persons, addition or deletion of sites, change in trial status, etc. Future challenges involve implementation of mandatory registration of
$\mathrm{BA} / \mathrm{BE}$ trials and results disclosure, along with other activities that would further increase public confidence and trust in clinical trials and their data.

Acknowledgments The CTRI has been set up with financial support from the Department of Science and Technology, Government of India, the Indian Council of Medical Research, Ministry of Health and Family Welfare and World Health Organization, New Delhi, India. The CTRI website is hosted by the National Informatics Centre Services Inc., New Delhi, India. The authors acknowledge the IT support provided by Mr. Yashmin Panchal, Research Scientist (Gr. II), Clinical Trials Registry - India, in the development and maintenance of the CTRI software application. No funding has been received for the preparation of this manuscript. The authors have no conflicts of interest that are directly relevant to the content of this review. 


\section{References}

1. Tharyan P, Ghersi D. Registering clinical trials in India: a scientific and ethical imperative. Natl Med J India. 2008;21(1):31-4.

2. Pandey A, Aggarwal AR, Seth SD, et al. Clinical Trials RegistryIndia: raising the veil. Natl Med J India. 2010;23(3):187-8.

3. Pandey A, Aggarwal AR, Maulik M, et al. Clinical trial registration gains momentum in India. Indian J Med Res. 2009; 130(1):85-6.

4. Pandey A, Aggarwal AR, Seth SD, et al. Clinical Trials RegistryIndia: redefining the conduct of clinical trials. Indian $\mathrm{J}$ Cancer. 2008;45(3):79-82.

5. Registration of clinical trials. http://www.cdsco.nic.in/clinical_ trial.htm. Accessed on 9th Jan 2013.
6. Tharyan P, George AT, Kirubakaran R, et al. Reporting of methods was better in the Clinical Trials Registry-India than in Indian journal publications. J Clin Epidemiol. 2013;66(1):10-22.

7. George B. CTRI-clicking to greater transparency and accountability. Perspect Clin Res. 2012;3:122-4.

8. Pandey A, Aggarwal A, Seth SD, et al. Strengthening ethics in clinical research. Indian J Med Res. 2011;133:339-40.

9. Satyanarayna K, Sharma A, Parikh P, et al. Statement on publishing clinical trials in Indian biomedical journals. Indian J Med Res. 2008;127(2):104-5.

10. De Angelis C, Drazen JM, Frizelle FA, et al. Clinical trial registration: a statement from the International Committee of Medical Journal Editors. N Engl J Med. 2004;351(12):1250-1. 